\title{
Modulating spontaneous brain activity using repetitive transcranial magnetic stimulation
}

\author{
Ysbrand D van der Werf ${ }^{1,2,3^{*}}$, Ernesto J Sanz-Arigita ${ }^{4,5}$, Sanne Menning ${ }^{6}$, Odile A van den Heuvel ${ }^{3,7}$
}

\begin{abstract}
Background: When no specific stimulus or task is presented, spontaneous fluctuations in brain activity occur. Brain regions showing such coherent fluctuations are thought to form organized networks known as 'resting-state' networks, a main representation of which is the default mode network. Spontaneous brain activity shows abnormalities in several neurological and psychiatric diseases that may reflect disturbances of ongoing thought processes. Information about the degree to which such spontaneous brain activity can be modulated may prove helpful in the development of treatment options. We investigated the effect of offline low-frequency rTMS on spontaneous neural activity, as measured with fMRI, using a sequential independent-component-analysis and regression approach to investigate local changes within the default mode network.

Results: We show that rTMS applied over the left dorsolateral prefrontal cortex results in distal changes of neural activity, relative to the site of stimulation, and that these changes depend on the patterns of brain network activity during 'resting-state'.

Conclusions: Whereas the proximal changes may reflect the off-line effect of direct stimulation of neural elements, the distal changes likely reflect modulation of functional connectivity.
\end{abstract}

\section{Background}

Brain activity underlying unconstrained thought can be visualized as resting-state networks [1-3]. Resting-state networks in health and disease are the topic of intensive investigation, but as yet little is known about factors that affect their appearance. The most well-known restingstate network, the default mode network (DMN) consists of concurrent activation of the medial prefrontal, the medial parietal and lateral parietal areas, in combination with medial and lateral temporal cortices [4]. This activity shows systematic deactivations during cognitive task performance that appear to be task-relevant [5,6]. Other functions ascribed to the DMN include introspection, memory processes and mind-wandering, although part of the activity is also accounted for by non-cognitive functions. It is not well known if the areas involved in the DMN contribute to a unified and general function or whether they represent separate contributions to ongoing

\footnotetext{
* Correspondence: y.van.der.werf@nin.knaw.nl

'Sleep and Cognition, Netherlands Institute for Neurosciences, an institute of the Royal Netherlands Academy of Arts and Sciences, Meibergdreef 47 ,

1105BA Amsterdam, The Netherlands

Full list of author information is available at the end of the article
}

thought. The fluctuating activity of the DMN seems to be controlled at least in part by different networks and regions $[7,8]$. The fluctuations of the DMN and other resting state networks occur both spontaneously and in relation to mental activity. It is as yet little known inhowfar external stimulation of the brain changes the resting state activity. Repetitive Transcranial Magnetic Stimulation (rTMS) is a tool to non-invasively and painlessly stimulate the brain [9]; depending on the stimulation parameters, the effect of a train of pulses will either facilitate or inhibit the activity of a neural ensemble $[10,11]$. The effects of rTMS outlast the period of stimulation and, depending on the parameters used, may last up to approximately half an hour or longer. Such stimulation has been shown to result in robust changes in various aspects of brain functioning, such as neuroransmitter release, task-related brain activity, motor output and behavioural indices [12-16]. Stimulation over the dorsolateral prefrontal cortex is an often used and potent modulator of both brain activity and task performance [17-23]. To our knowledge, however, no studies have as yet investigated the effect of dorsolateral rTMS on resting state brain activity. 
We here use a single session of low-frequency rTMS treatment on the dorsal lateral prefrontal cortex to study resting state brain activity in healthy subjects. We hypothesized that rTMS, applied locally over the left dorsal lateral prefrontal cortex, would alter the strength or the spatial distribution, or both, of spontaneous brain activity. We analysed the effects of the rTMS treatment on the most well-known of the resting state brain networks, the default mode network. This network is the most robust of the resting state networks under taskfree conditions. It is especially relevant for psychiatric disorders, especially depression, in which dorsal lateral rTMS appears effective [24].

We compared the stimulation with a sham stimulation to control for peripheral effects of stimulation and the placebo effects of the treatment; the sham condition consisted of tilting the coil 90 degerees, such that it rested on the head with its edge. The bone conduction of the clicking sound would then be comparable to the real stimulation. We took care to blind the subjects by not showing the angulation of the coil and explaining that the sensation of stimulation could vary from session to session. The choice of a placebo condition is notoriously hard in rTMS research since treatment of 'inactive areas' to show specificity of the stimulation may sometimes lead to unblinding of the subjects or to unwanted effects due to passive or active spreading of the activity (e.g. [25]).

\section{Results}

\section{Resting motor thresholds}

We determined resting motor thresholds in our subjects on both days of testing. No within-subject differences between thresholds were found between conditions, resulting in stimulation intensities (at 90\% of resting motor threshold) that were not different between conditions.

\section{RSN networks}

Our analysis yielded 41 resting-state networks. Of these networks, we selected the meaningful components that corresponded to networks described earlier, i.e. the 7 components shown in figure $1[4,26]$. We did not find an RSN corresponding to a 'working memory network' in the left hemisphere in this study [26], although we did observe its right-hemisphere homologue.

For this analysis, we focused on the topmost component, representing the DMN. Our results indicate that this component comprises the well-known regions, i.e. the medial prefrontal cortex, precuneus and lateral parietal cortex. In accord with several studies, IC4 also encompassed the hippocampus proper and middle temporal gyrus (Figure 2). We reconstructed the groupwise means (i.e. sham and rTMS conditions: $n=10$ each)

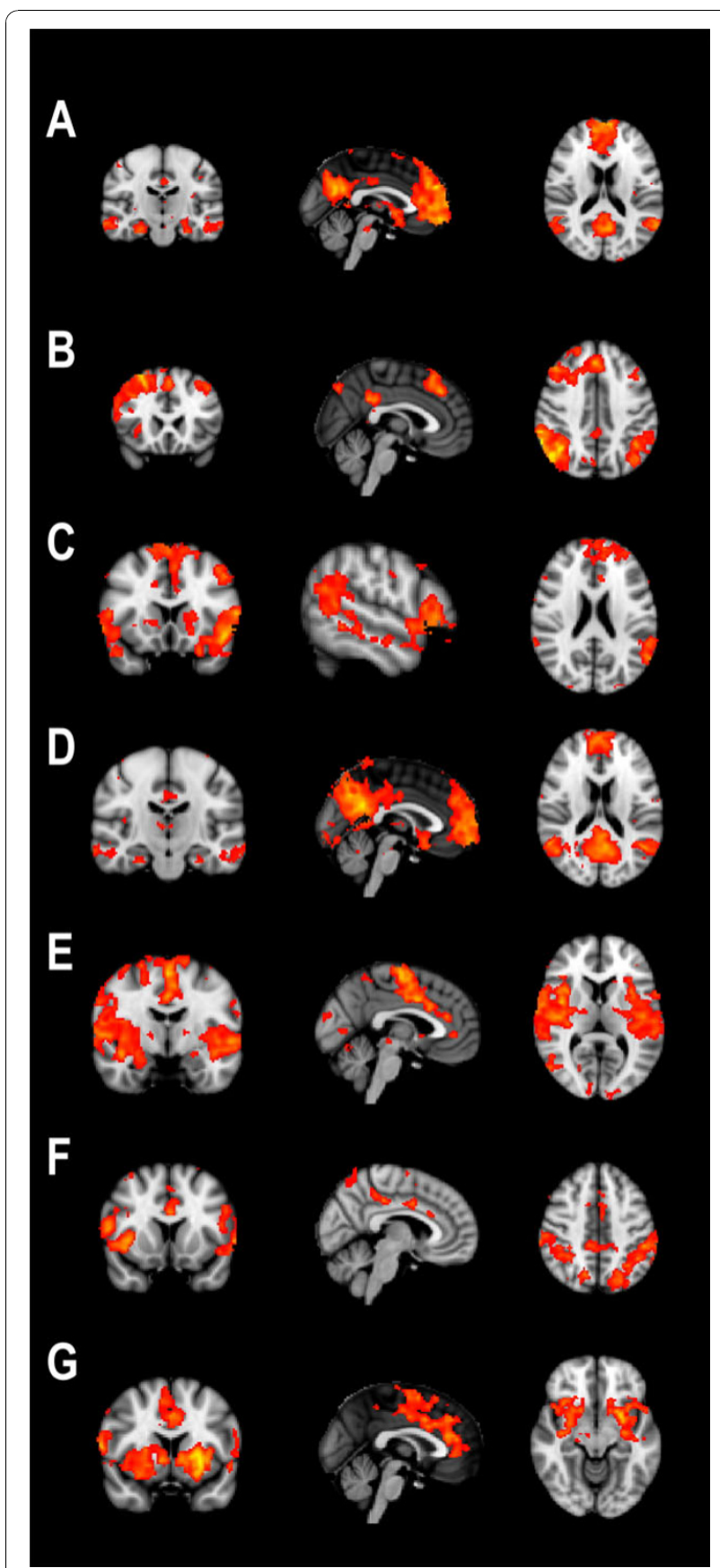

Figure 1 Meaningful components from the independent component analysis across the $\mathbf{2 0}$ resting state acquisitions. The topmost component represents the DMN used for this analysis. Note that component $\mathrm{D}$ also represents the $\mathrm{DMN}$, but slightly more posteriorly weighted.

and observed the same network consisting of the same regions for the two stimulation conditions separately, with the notable exception of the hippocampus and lateral temporal cortex that were not observed in the low-frequency rTMS condition (Figure 2). Upon formal testing, contrasting condition using session as a within- 


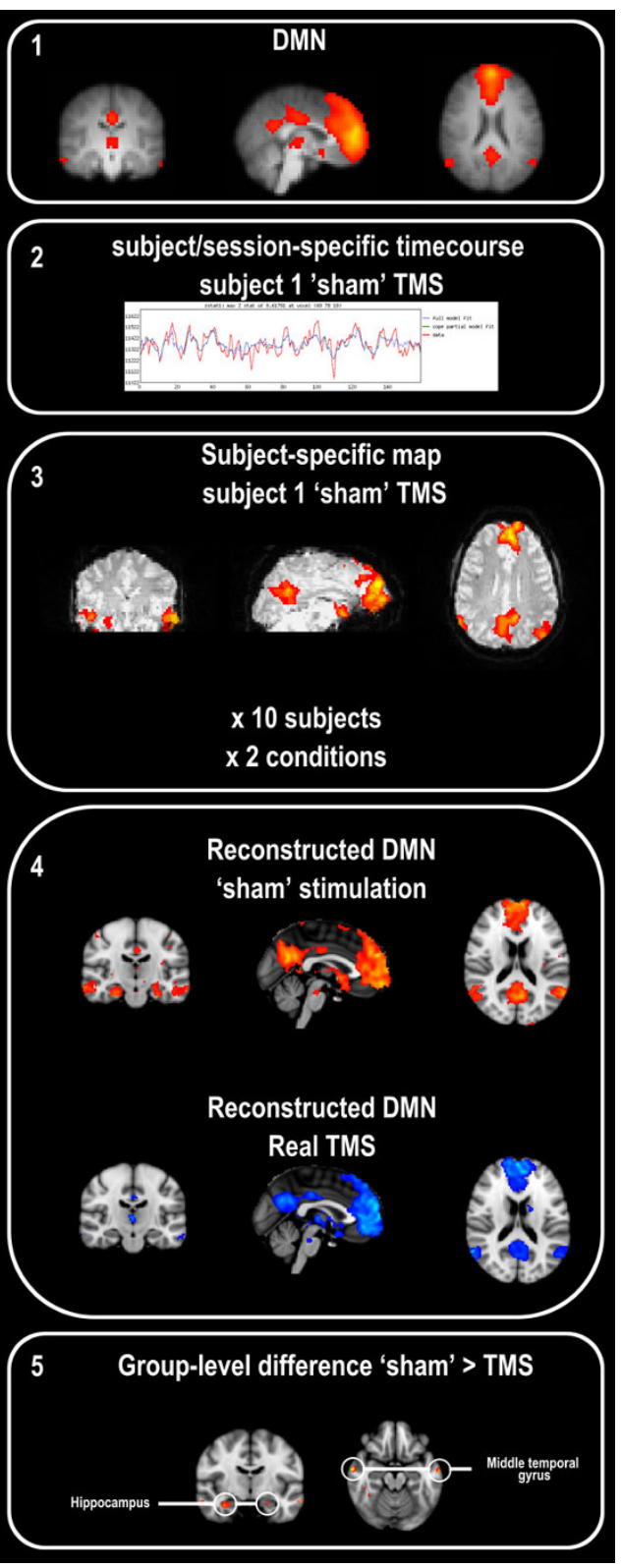

Figure 2 Step-wise procedure and end result of the regression method. From the independent component representing the DMN, obtained with spatiotemporal group ICA (1), we used the subjectand-session specific timecourses (2) to reconstruct subject-specific 3D maps for the 10 subjects*2 sessions (3). As a verification, we calculated the group means of the sham and rTMS groups separately ('reconstructed IC4') and observed that the DMNcharacteristic pattern of regional co-activations occurred in both groups, with an additional co-activation of the hippocampal and lateral temporal cortices after sham stimulation but not real stimulation (4). Upon formal testing, using groupwise within-subject component general linear modelling, the lateral temporal regions differed significantly between conditions, such that their activation was reduced after rTMS. The image is thresholded at $z>2.3$, showing the extent of the reductions and subthreshold reductions in the bilateral hippocampus (5). The reverse contrast showed an increase in activation of the right caudate nucleus (not shown).
Table 1 Brain regions showing reduced or increased activity after rTMS

\begin{tabular}{cccccc}
\hline Contrast & Zmax & $\mathbf{x}$ & $\mathbf{y}$ & $\mathbf{z}$ & Area \\
\hline sham $>$ rTMS & 3.23 & -64 & -8 & -16 & Left middle temporal gyrus \\
& 3.54 & 62 & -6 & -18 & right middle temporal gyrus \\
sham $>$ rTMS & 3.28 & 14 & 16 & 8 & Right caudate nucleus \\
\hline
\end{tabular}

The left and right middle temporal gyrus appeared at $z>3.1$, using a search mask consisting of the distribution of the original component.

subject factor, we observed that the activations in the lateral temporal cortex were significantly stronger after 'sham' rTMS ( $\mathrm{z}>3.1$; Table 1$)$. Lowering the significance threshold to $\mathrm{z}>2.3$ in the regions that are part of the DMN in this analysis, the hippocampus proper showed reduced activation bilaterally (figure 1 ). In the reverse contrast, activation in the right caudate nucleus was seen after rTMS but not sham $(\mathrm{z}>3.1$; Table 1$)$.

\section{Discussion}

We here show changes of DMN activity upon treatment with inhibitory low-frequency rTMS intervention. The alterations in DMN strength occurred distal to the site of stimulation, i.e. prefrontal stimulation led to reductions in DMN activity bilaterally in the temporal lobes and an increase in the caudate nucleus. Previous reports have shown the existence of multiple constellations of simultaneously active areas in the human brain, that supposedly correspond to specific brain functions or processes [26]. The default mode network is the most well-known of these and can also be observed as inactivations during task performance, measured using BOLD fMRI or PET [4]. The DMN may correspond in part to introspection, reflection or spontaneous cognition in the absence of external stimulation, but also reflects processes unrelated to conscious cognition such as maintenance of functional integrity of brain networks or metabolic demands $[27,28]$. Several reports have shown that the hippocampal formation and adjacent medial temporal cortical structures are part of the DMN [29-31]. These areas are well-known for their role in semantic and episodic memory formation and retrieval as well as novelty detection.

We analysed the effects of rTMS treatment on the activity of the most important resting state network. We performed a within-component comparison of the two conditions, aimed at revealing differences at the level of the DMN. A different approach would be to investigate interactions between networks and their timing [32]. Such studies would allow to investigate the balance between different brain systems and the changes that rTMS may exert on such interactions. A limitation of this study is that the coil was placed over the left dorsolateral prefrontal cortex using the motor 'hotspot' as a landmark. Such a coil placement technique is more 
variable with respect to the underlying brain structures targeted than neuronavigation approaches using the individual MRI of the subjects [33]. On the other hand, the results presented here would be an underestimation, if anything, of the effect that more targeted interventions could have. At the same time, coil placement using head-based landmarks does make the technique more readily available outside of a specialized laboratory setting, and more easy to implement should the technique become a treatment option.

Repetitive TMS as a treatment may be relevant for psychiatric disorders such as depression, obsessive compulsive disorder or schizophrenia that are characterized by spontaneous intrusive thoughts. Indeed, several psychiatric disorders are characterized by abnormal brain activity in the resting state [34-37]. It would be of interest to modulate ongoing brain activity, offering either direct clinical benefit or a window of time in which a patient might be more receptive to other kinds of therapy, such as psychotherapy. Indeed, rTMS has been considered as a treatment option, most notably in major depression [24,38]. Since firstly, stimulating the dorsal lateral prefrontal cortex appears to benefit mood disturbances; and secondly, depression is associated with disturbed DMN activity, this raises the question of whether mood improvements after rTMS are associated with changes in the DMN, even though the area stimulated lies outside the DMN itself. Speculatively, the reduced activation in hippocampal and lateral temporal areas after rTMS over the dorsolateral prefrontal cortex may affect autobiographical or semantic memory retrieval, of importance for disturbed self-referential cognitive tendencies in psychiatric disease: depressed patients observing and re-appraising negative images, for example, showed a failure to suppress DMN activity in lateral temporal cortices, while even increasing activity in the medial temporal lobe [36]. Suppression of temporal lobe activity may thus underlie the beneficial effects of prefrontal rTMS on depressed mood, through an effect on spontaneous mental activity outlasting the duration of treatment.

\section{Conclusions}

Low-frequency rTMS over the left dorsolateral prefrontal cortex affects off-line resting-state brain activation. The intervention reduces RSN activity within the DMN. The reductions of activation occur in the temporal lobes that are distal from the area stimulated, suggesting an effect of rTMS on long-range functional connectivity.

\section{Methods}

Ten right-handed healthy controls (6 female; mean age 25.5 years) were entered in a cross-over design: two single session treatments (counterbalanced across the group) of low-frequency $(1 \mathrm{~Hz})$ rTMS versus sham for 20 minutes on the left dorsolateral prefrontal cortex. The coil was placed $5 \mathrm{~cm}$ anterior to the 'motor hotspot', i.e. the location where stimulation led to maximal motor responses in the contralateral hand. We determined the resting motor threshold at this 'motor hotspot' as that intensity at which 5 visible hand/finger responses could be evoked out of a series of 10 consecutive stimulations.

Repetitive TMS was applied using a hand-held figureof-eight TMS coil (Medtronic MagOption). Directly following the off-line rTMS treatment, 160 volumes were acquired of the brain in 'resting-state' with a 3T Philips Intera MRI (EPI, TR $2.30 \mathrm{sec}$., TE $30 \mathrm{msec}$., matrix $96 \times 96$ pixels, field of view $220 \times 220 \mathrm{~mm}$, flip angle $80^{\circ}$, 35 slices, slice thickness $3 \mathrm{~mm}$, in plane resolution $2.3 \times 2.3 \mathrm{~mm}$ ).

Imaging data were first converted from the original PAR/REC files to Analyze format using MRIcro (Chris Rorden). We then used pre-processing and statistics using tools implemented in FMRIB's Software Library (FSL, http://www.fmrib.ox.ac.uk/fsl) as follows: the functional MR images were motion-corrected using MCFLIRT and nonbrain tissue was removed with BET. The images were spatially smoothed using a Gaussian kernel of six mm full-width-at-half-maximum (FWHM) and a high-pass temporal filtering was applied (Gaussian-weighted least-squares straight line fitting, with sigma $=100 \mathrm{~s}$ ). The functional scan was then aligned to the subject's high resolution T1-weighted image, and subsequently to the MNI152 standard through affine linear registration as implemented in FLIRT.

After preprocessing, a unique 4D data set was created by concatenating all the individual data. This concatenated fMRI data set was decomposed using ICA as part of Multivariate Exploratory Linear Optimized Decomposition into Independent Components (MELODIC) to identify homogeneous patterns of brain activity in our subjects [39]. The analysis used automatic estimation of dimensionality to control the number of components reported. We extracted the top meaningful components ranked according to the amount of explained variance, each representing statistically independent resting state networks. Components were deemed 'meaningful' on the basis of visual inspection of the spectra and spatial distribution: e.g. networks consisting of artefacts such as ventricular, white matter and brain circumferential activations were excluded, as were components showing irregular frequency spectra. Components obtained were compared to those reported earlier for validation. Only those corresponding in spatial distribution to components reported by Damoiseaux et al. were considered [26]. For the ensuing analysis we selected the topmost meaningful component, which represents the default 
mode network (i.e. component 4). Using the individual timecourses of the multi-session concatenated ICA components across subjects and conditions, we reconstructed subject-specific maps in native stereotaxic space using FMRIB's Improved Linear Model (FILM) in FMRI Expert Analysis Tool (FEAT). We then made group comparisons (using FMRIB's Local Analysis of Mixed Effects (FLAME)) to contrast the real and the 'sham' rTMS condition in a within-subject design; all results of the group analysis were warped to MNI standard space. We focused on the strongest meaningful component; this component corresponded to the default mode network (DMN).

BOLD signal contrasts for the comparison of the two groups were considered significant at a threshold of $\mathrm{z}>3.1$ ( $\mathrm{p}<0.001$ ); to be sensitive to small but meaningful changes we conducted a directed search, i.e. within a mask consisting of the regions encompassed by the IC4.

\section{Abbreviations}

BOLD: blood oxygen level dependent; DMN: default mode network; FMRI: functional magnetic resonance imaging; IC: independent component; PET: positron emission tomography; RSN: resting state network; (r)TMS: (repetitive) transcranial magnetic stimulation;

\section{Acknowledgements}

The authors thank Richard van Dyck for financial support, Dick Veltman for conceptual advice, Maloe Hulst and Helene van Gorsel for contribution to data collection, Aart Nederveen for technical support, and Eus van Someren for support in equipment. This work was supported by a VENI-grant ZonMW 916.86.038 (OAvdH) from the Dutch Organization for Scientific Research (Nederlandse Organisatie voor Wetenschappelijk Onderzoek, NWO).

\footnotetext{
Author details

${ }^{1}$ Sleep and Cognition, Netherlands Institute for Neurosciences, an institute of the Royal Netherlands Academy of Arts and Sciences, Meibergdreef 47, 1105BA Amsterdam, The Netherlands. ${ }^{2}$ Clinical Neurophysiology, VU University medical center, PO Box 7057 1007MB Amsterdam, The Netherlands. ${ }^{3}$ Dept Anatomy and Neurosciences, VU University medical center, PO Box 7057 1007MB Amsterdam, The Netherlands. ${ }^{4}$ Radiology, VU University medical center, PO Box 7057 1007MB Amsterdam, The Netherlands. ${ }^{5}$ Dept Radiology, CITA-Alzheimer Foundation, Parque Tecnológico de San Sebastián, P o Mikeletegi 61, 20009 Donostia-San Sebastián, Spain. ${ }^{6}$ Dept Psychosocial Research and Epidemiology, Netherlands Cancer Institute, Plesmanlaan 121, 1066CX Amsterdam, The Netherlands. 'Psychiatry, VU University medical center, PO Box 7057 1007MB Amsterdam, The Netherlands.
}

\section{Authors' contributions}

YVW designed the study, performed data acquisition and wrote the manuscript; EJSA supervised the analysis and co-wrote the manuscript; SM analyzed and interpreted the data; OAVDH designed the study, performed data acquisition and co-wrote the manuscript. All authors read and approved the final manuscript.

Received: 4 February 2010 Accepted: 10 November 2010 Published: 10 November 2010

\section{References}

1. Gusnard DA, Akbudak E, Shulman GL, Raichle ME: Medial prefrontal cortex and self-referential mental activity: relation to a default mode of brain function. Proc Natl Acad Sci USA 2001, 98:4259-4264.
2. Raichle ME, MacLeod AM, Snyder AZ, Powers WJ, Gusnard DA, Shulman GL: A default mode of brain function. Proc Natl Acad Sci USA 2001, 98:676-682.

3. Horovitz SG, Braun AR, Carr WS, Picchioni D, Balkin TJ, Fukunaga M, Duyn JH: Decoupling of the brain's default mode network during deep sleep. Proc Natl Acad Sci USA 2009, 106:11376-11381.

4. Smith SM, Fox PT, Miller KL, Glahn DC, Fox PM, Mackay CE, Filippini N, Watkins KE, Toro R, Laird AR, Beckmann CF: Correspondence of the brain's functional architecture during activation and rest. Proc Natl Acad Sci USA 2009, 106:13040-13045.

5. Eichele T, Debener S, Calhoun VD, Specht K, Engel AK, Hugdahl K, von Cramon DY, Ullsperger M: Prediction of human errors by maladaptive changes in event-related brain networks. Proc Natl Acad Sci USA 2008, 105:6173-6178.

6. Li CS, Yan P, Bergquist KL, Sinha R: Greater activation of the "default" brain regions predicts stop signal errors. Neuroimage 2007, 38:640-648

7. Fox MD, Snyder AZ, Vincent JL, Corbetta M, Van Essen DC, Raichle ME: The human brain is intrinsically organized into dynamic, anticorrelated functional networks. Proc Natl Acad Sci USA 2005, 102:9673-9678.

8. Sridharan D, Levitin DJ, Menon V: A critical role for the right fronto-insular cortex in switching between central-executive and default-mode networks. Proc Natl Acad Sci USA 2008, 105:12569-12574.

9. Kobayashi M, Pascual-Leone A: Transcranial magnetic stimulation in neurology. Lancet Neurol 2003, 2:145-156.

10. Chen R: Studies of human motor physiology with transcranial magnetic stimulation. Muscle Nerve Supp/ 2000, 9:S26-32.

11. Fitzgerald PB, Fountain S, Daskalakis ZJ: A comprehensive review of the effects of rTMS on motor cortical excitability and inhibition. Clin Neurophysiol 2006, 117:2584-2596.

12. Chouinard PA, Van Der Werf YD, Leonard G, Paus T: Modulating neural networks with transcranial magnetic stimulation applied over the dorsal premotor and primary motor cortices. J Neurophysiol 2003, 90:1071-1083.

13. Van Der Werf YD, Paus T: The neural response to transcranial magnetic stimulation of the human motor cortex. I. Intracortical and corticocortical contributions. Exp Brain Res 2006, 175:231-245.

14. Ko JH, Monchi O, Ptito A, Petrides M, Strafella AP: Repetitive Transcranial Magnetic Stimulation of Dorsolateral Prefrontal Cortex Affects Performance of the Wisconsin Card Sorting Task during Provision of Feedback. Int J Biomed Imaging 2008, 2008:143238.

15. Strafella AP, Paus T, Fraraccio $M$, Dagher A: Striatal dopamine release induced by repetitive transcranial magnetic stimulation of the human motor cortex. Brain 2003, 126:2609-2615.

16. Oliveri M, Torriero S, Koch G, Salerno S, Petrosini L, Caltagirone C: The role of transcranial magnetic stimulation in the study of cerebellar cognitive function. Cerebellum 2007, 6:95-101.

17. Barrett J, Della-Maggiore V, Chouinard PA, Paus T: Mechanisms of action underlying the effect of repetitive transcranial magnetic stimulation on mood: behavioral and brain imaging studies. Neuropsychopharmacology 2004, 29:1172-1189.

18. Rounis E, Stephan KE, Lee L, Siebner HR, Pesenti A, Friston KJ, Rothwell JC, Frackowiak RS: Acute changes in frontoparietal activity after repetitive transcranial magnetic stimulation over the dorsolateral prefrontal cortex in a cued reaction time task. J Neurosci 2006, 26:9629-9638.

19. Rounis E, Yarrow K, Rothwell JC: Effects of rTMS conditioning over the fronto-parietal network on motor versus visual attention. J Cogn Neurosci 2007, 19:513-524.

20. Strafella AP, Paus T, Barrett J, Dagher A: Repetitive transcranial magnetic stimulation of the human prefrontal cortex induces dopamine release in the caudate nucleus. J Neurosci 2001, 21:RC157.

21. Vanderhasselt MA, De Raedt R, Baeken C, Leyman L, Clerinx P, D'Haenen H: The influence of rTMS over the right dorsolateral prefrontal cortex on top-down attentional processes. Brain Res 2007, 1137:111-116.

22. Vanderhasselt MA, De Raedt R, Baeken C, Leyman L, D'Haenen H: The influence of rTMS over the right dorsolateral prefrontal cortex on intentional set switching. Exp Brain Res 2006, 172:561-565.

23. Paus T, Castro-Alamancos MA, Petrides M: Cortico-cortical connectivity of the human mid-dorsolateral frontal cortex and its modulation by repetitive transcranial magnetic stimulation. Eur J Neurosci 2001, 14:1405-1411.

24. O'Reardon JP, Solvason HB, Janicak PG, Sampson S, Isenberg KE, Nahas Z, McDonald WM, Avery D, Fitzgerald PB, Loo C, et al: Efficacy and Safety of 
Transcranial Magnetic Stimulation in the Acute Treatment of Major Depression: A Multisite Randomized Controlled Trial. Biol Psychiatry 2007, 62(11):1208-16.

25. Helmich RC, Siebner HR, Bakker M, Munchau A, Bloem BR: Repetitive transcranial magnetic stimulation to improve mood and motor function in Parkinson's disease. J Neurol Sci 2006, 248:84-96.

26. Damoiseaux JS, Rombouts SA, Barkhof F, Scheltens P, Stam CJ, Smith SM, Beckmann CF: Consistent resting-state networks across healthy subjects. Proc Natl Acad Sci USA 2006, 103:13848-13853.

27. Fukunaga M, Horovitz SG, de Zwart JA, van Gelderen P, Balkin TJ, Braun AR, Duyn JH: Metabolic origin of BOLD signal fluctuations in the absence of stimuli. J Cereb Blood Flow Metab 2008, 28:1377-1387.

28. Larson-Prior LJ, Zempel JM, Nolan TS, Prior FW, Snyder AZ, Raichle ME: Cortical network functional connectivity in the descent to sleep. Proc Natl Acad Sci USA 2009, 106:4489-4494.

29. Damoiseaux JS, Beckmann CF, Arigita EJ, Barkhof F, Scheltens P, Stam CJ, Smith SM, Rombouts SA: Reduced resting-state brain activity in the "default network" in normal aging. Cereb Cortex 2008, 18:1856-1864.

30. Filippini N, MacIntosh BJ, Hough MG, Goodwin GM, Frisoni GB, Smith SM, Matthews PM, Beckmann CF, Mackay CE: Distinct patterns of brain activity in young carriers of the APOE-epsilon4 allele. Proc Natl Acad Sci USA 2009, 106:7209-7214.

31. Toro R, Fox PT, Paus T: Functional coactivation map of the human brain Cereb Cortex 2008, 18:2553-2559.

32. Jafri MJ, Pearlson GD, Stevens M, Calhoun VD: A method for functional network connectivity among spatially independent resting-state components in schizophrenia. Neuroimage 2008, 39:1666-1681.

33. Sack AT, Cohen Kadosh R, Schuhmann T, Moerel M, Walsh V, Goebel R: Optimizing functional accuracy of TMS in cognitive studies: a comparison of methods. J Cogn Neurosci 2009, 21:207-221.

34. Garrity AG, Pearlson GD, McKiernan K, Lloyd D, Kiehl KA, Calhoun VD: Aberrant "default mode" functional connectivity in schizophrenia. Am J Psychiatry 2007, 164:450-457.

35. Kwon JS, Jang JH, Choi JS, Kang DH: Neuroimaging in obsessivecompulsive disorder. Expert Rev Neurother 2009, 9:255-269.

36. Sheline YI, Barch DM, Price JL, Rundle MM, Vaishnavi SN, Snyder AZ, Mintun MA, Wang S, Coalson RS, Raichle ME: The default mode network and self-referential processes in depression. Proc Natl Acad Sci USA 2009, 106:1942-1947.

37. Whitfield-Gabrieli S, Thermenos HW, Milanovic S, Tsuang MT, Faraone SV, McCarley RW, Shenton ME, Green Al, Nieto-Castanon A, LaViolette P, et al: Hyperactivity and hyperconnectivity of the default network in schizophrenia and in first-degree relatives of persons with schizophrenia. Proc Natl Acad Sci USA 2009, 106:1279-1284

38. George MS, Nahas Z, Molloy M, Speer AM, Oliver NC, Li XB, Arana GW Risch SC, Ballenger JC: A controlled trial of daily left prefrontal cortex TMS for treating depression. Biol Psychiatry 2000, 48:962-970.

39. Beckmann CF, Smith SM: Tensorial extensions of independent component analysis for multisubject FMRI analysis. Neuroimage 2005, 25.294-311.

doi:10.1186/1471-2202-11-145

Cite this article as: van der Werf et al:: Modulating spontaneous brain activity using repetitive transcranial magnetic stimulation. $B M C$ Neuroscience 2010 11:145.

\section{Submit your next manuscript to BioMed Central and take full advantage of:}

- Convenient online submission

- Thorough peer review

- No space constraints or color figure charges

- Immediate publication on acceptance

- Inclusion in PubMed, CAS, Scopus and Google Scholar

- Research which is freely available for redistribution

Submit your manuscript at www biomedcentral com/submit
C Biomed Central 